\title{
The Effect of Spark Plasma Sintering on Microstructure Evolution in Thermoelectric Materials
}

Xiaomi Zhang ${ }^{1}$, Zhong-Zhen Luo ${ }^{2,3}$, Sumanta Sarkar ${ }^{2}$, Mercouri G. Kanatzidis ${ }^{2,4}$ and Vinayak P. Dravid $^{1,5}$

1. Department of Materials Science and Engineering, Northwestern University, Evanston, USA.

2. Department of Chemistry, Northwestern University, Evanston, USA.

3. School of Materials Science and Engineering, Nanyang Technological University, Singapore.

4. Materials Science Division, Argonne National Laboratory, Argonne, USA.

5. NUANCE Center, Northwestern University, Evanston, USA.

Thermoelectric materials have attracted particular attention thanks to their unique ability to convert waste heat to electrical energy with outstanding stability. The efficiency of thermoelectrics is evaluated by the "figure of merit" $Z T={ }^{S^{2} \sigma T} / \mathbb{R}\left(\kappa \rrbracket_{e}+\kappa_{l}\right)^{*}$ in which $S$ is the Seebeck coefficient, $\sigma$ is electrical conductivity, $\mathrm{T}$ is absolute temperature, and $\kappa_{e}$ and $\kappa_{l}$ are electrical and lattice thermal conductivities.

Many researchers have demonstrated thermoelectric performance improvement by reducing the lattice thermal conductivity via addition of point defects[1], dislocations[2], grain boundaries[3, 4] and nanoscale precipitates[5] into a common thermoelectric matrix material. Spark Plasma Sintering (SPS) is a pressure assisted, current activated, sintering technique commonly used for many thermoelectric materials. SPS can provide materials with high density, small grain size, and large grain boundary areas [6]. However, recent studies have shown temperature and current inhomogeneities throughout the specimen during the sintering process. How these inhomogeneities affect the thermoelectric microstructure has not yet been reviewed systematically [6], and miniscule microstructure changes such as grain boundary inhomogeneity or second phase generation during the SPS process can affect carrier or phonon transport, therefore changing the performance of thermoelectrics unexpectedly.

The presentation will cover the microstructure and thermoelectric performance changes of lead chalcogenide thermoelectric materials during the SPS process, with two high performance systems as an example, n-type Sb-doped PbTe alloyed with GeTe (PbTe-GeTe) and p-type Na-doped PbTe alloyed with $\mathrm{BaTe}$ and $\mathrm{CaTe}\left(\mathrm{PbTe}-\mathrm{Ba}_{0.5} \mathrm{Ca}_{0.5} \mathrm{Te}\right)$. The ingot samples were prepared using a solid-state reaction method and then annealed for two days at $573 \mathrm{~K}$ for PbTe-GeTe and at $873 \mathrm{~K}$ for PbTe- $\mathrm{Ba}_{0.5} \mathrm{Ca}_{0.5} \mathrm{Te}$. The ingots were hand-ground and SPS-ed at $823 \mathrm{~K}$ for five minutes at $40 \mathrm{MPa}$. Both ingot systems appear to have microscale second phases that are deficient in $\mathrm{Pb}$, and each individually rich in $\mathrm{Ba}, \mathrm{Te}(\mathrm{PbTe}-$ $\mathrm{Ba}_{0.5} \mathrm{Ca}_{0.5} \mathrm{Te}$ ) and $\mathrm{Ge}$, Te (PbTe-GeTe). However, in the SPS-ed samples of both systems, nanoscale precipitates exist and no microscale second phases are observed. We will discuss how different parameters in the SPS process can change the microstructure of thermoelectrics, especially their composition, crystal structure, and grain boundary structures.

SPS can significantly modify the microstructure of thermoelectric materials in previously unforeseen ways. Studying the microstructure changes during the SPS process can reveal pivotal information in assessing structure-property relationships within thermoelectrics. These details can allow the precise tuning of thermoelectric processing to achieve vast performance improvements. This presentation will also cover detailed analyses of the evolution of material's crystal structure, microstructure and 
thermoelectric properties induced during the SPS process [7].

\section{References:}

1. He, J. et al, J Am Chem Soc 135(12) (2013) p. 4624.

2. Chen, Z. et al, Nature communications 8 (2017) p. 13828.

3. Biswas, K. et al, Nature 489(7416) (2012) p. 414.

4. Wu, D. et al, Energy \& Environmental Science 8(7) (2015) p. 2056.

5. Girard, S.N. et al, Journal of the American Chemical Society 133(41) (2011) p. 16588.

6. Munir, Z. et al, Journal of Materials Science 41(3) (2006) p. 763.

7. This work was supported by the Department of Energy, Office of Science, Basic Energy Sciences under Grant DE-SC0014520, made use of the EPIC facility of Northwestern University's NUANCE Center, which has received support from the Soft and Hybrid Nanotechnology Experimental (SHyNE) Resource (NSF ECCS1542205); the MRSEC program (NSF DMR-1121262) at the Materials Research Center; the International Institute for Nanotechnology (IIN); the Keck Foundation; and the State of Illinois, through the IIN.

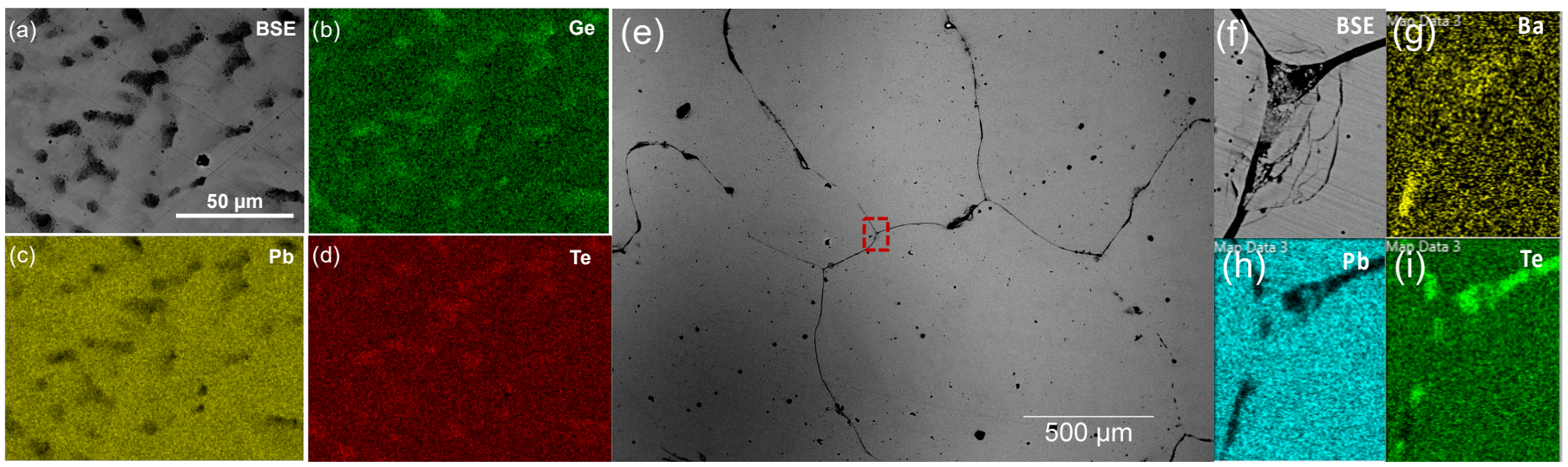

Figure 1. (a)Backscattered electron (BSE) image and (b) Ge, (c) Pb and (d) Te Energy Dispersive X-ray Spectroscopy (EDS) elemental maps for ingot $\left(\mathrm{Pb}_{0.988} \mathrm{Sb}_{0.012} \mathrm{Te}\right)_{0.87}(\mathrm{GeTe})_{0.13}$. (e) Low magnification and (f) high magnification BSE images and $(\mathrm{g}) \mathrm{Ba}$, (h) $\mathrm{Pb}$, (i) Te EDS elemental maps for ingot $\left(\mathrm{Pb}_{0.98} \mathrm{Na}_{0.02} \mathrm{Te}\right)_{0.99}-\left(\mathrm{Ba}_{0.5} \mathrm{Ca}_{0.5} \mathrm{Te}\right)_{0.01}$.
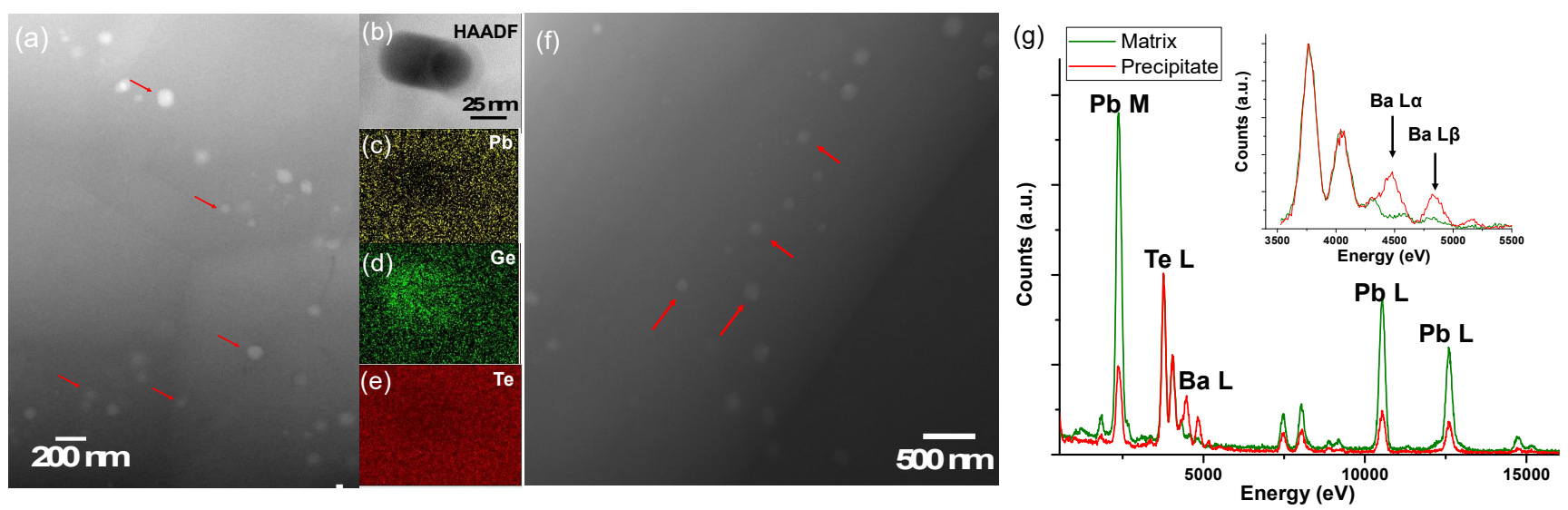

Figure 2. (a) ADF STEM image of SPS-ed $\left(\mathrm{Pb}_{0.988} \mathrm{Sb}_{0.012} \mathrm{Te}\right)_{0.87}-(\mathrm{GeTe})_{0.13}$. Nanoscale precipitates are selectively indicated by red arrows. (b)HAADF STEM image of a representative precipitate. (c)-(e) corresponding EDS elemental maps for $\mathrm{Pb}, \mathrm{Ge}$ and $\mathrm{Te}$, showing the precipitate is Ge rich and Te deficient. (f) ADF STEM image of SPS-ed $\left(\mathrm{Pb}_{0.98} \mathrm{Na}_{0.02} \mathrm{Te}\right)_{0.99}-\left(\mathrm{Ba}_{0.5} \mathrm{Ca}_{0.5} \mathrm{Te}\right)_{0.01}$. (g) Representative EDS taken from both matrix (green solid line) and nanoscale precipitate (red solid line). The inset spectra highlight differences in $\mathrm{Ba} \mathrm{L}$ peak intensities. The precipitate has higher Ba concentration than the matrix. 